\title{
ON PASSIVE MOTION OF THE ARMS FOR A WALKING PLANAR BIPED
}

\author{
BASSEL KADDAR, YANNICK AOUSTIN and CHRISTINE CHEVALLEREAU \\ LUNAM IRCCYN, UMR 6597 C.N.R.S \\ Ecole Centrale de Nantes, Université de Nantes, BP 92101 \\ 1 rue de la Nö̈, 44321 Nantes cedex 03, France \\ E-mail: Bassel.Kaddar@irccyn.ec-nantes.fr,Yannick.Aoustin@irccyn.ec-nantes.fr \\ Christine.Chevallereau@irccyn.ec-nantes.fr
}

\begin{abstract}
The energetic effects of passive motion of the arms and addition of torsion springs to the arms joints of a nine-link planar bipedal robot are studied for walking gaits. Only the locomotor system of the biped is actuated. Starting from a cyclic optimal motion of the biped with the arms stuck to the trunk, we explored the existence of a passive motion of the arms produced by the dynamics of the locomotor system of the biped. Multiples solutions including large amplitude of arms motion exist if the duration of walking step corresponds to the natural period of arms oscillation. The effect of torsion springs on the natural period and the cost functional is explored. Finally, costs functional of reference trajectories and trajectories with passive motions of the arms are compared.
\end{abstract}

\subsection{Introduction}

Several studies are done on the definition of walking gaits [1, 2]. For many researchers, trajectories of walking are achieved by using optimization [3]. Bipedal walking gaits with arms are also studied. Many of these studies interest with the generation of upper body motion aiming at improving the motion stability of the robot biped [4]; likely by the compensation of yaw moment [5]. Few studies and results are available to describe the effects of arms on bipedal walking gaits especially on the energy consumption. Aoustin and Formal'skii studied the optimal arm swinging of a planar biped [6]. Walking gaits are ballistic and torques are applied only during the impact. They showed that for a given period of the walking gait step and a length of the step, there is optimal amplitude of arm swing for which a cost functional is minimum. The effect of arms motions on the energy consumption, where the robot biped is fully actuated or only joints of the locomotor system are actuated, has not yet been shown. 
The scope of this work is to investigate the dynamical effects of a passive motion of the arms on the energy consumption of a planar bipedal robot during its walking gait.

We first look for if a passive motion of the arms, due to the dynamic of actuated locomotor system, may exist for the walking gait. It is shown that large amplitude passive motion of the arms can be obtained for only an appropriate duration of the walking step. The introduction of torsion springs into shoulder joints is also studied to enable large passive motion of the arms with various walking periods. Finally, we show the dynamical effects of this passive motion of the arms on the energy consumption during walking of a planar bipedal robot.

\subsection{Presentation of the biped and walking gait}

The biped under study, presented in the figure 1, is a nine-link planar biped composed of two identical legs, two identical one-link arms and a torso. Each leg consists of a femur, a tibia and a rigid foot. All joints are revolute and have only one degree of freedom such that they can only move in the sagittal plane. Our biped is 2D equivalent of humanoid robot HYDROiD [7]. Desired gaits consist of single support phases separated by instantaneous and perfectly inelastic contact on the ground. This contact is supposed to be without impact.

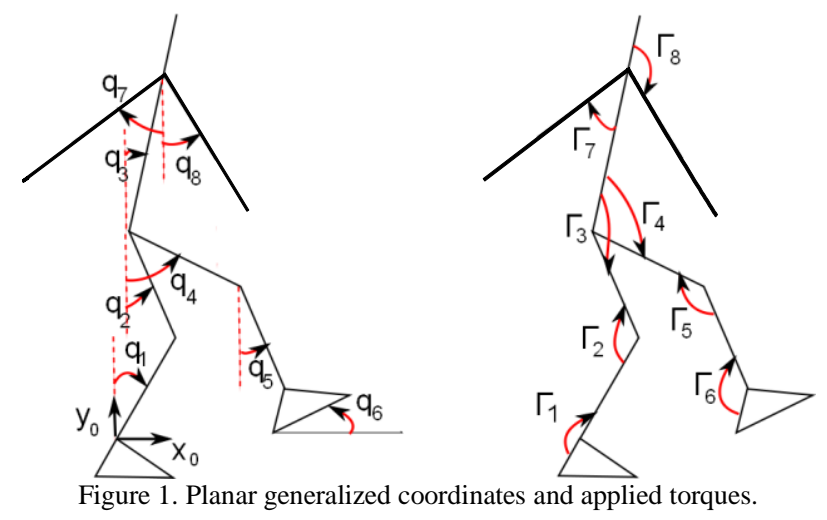

The walking step starts with a single support phase and ends with the contact of the swing foot on the ground where the feet change their role, i.e. the stance foot becomes the swing foot and vice versa. The instantaneous contact on the ground is supposed to be with a flat foot and there is no rotation on the heel or toe of stance foot during swinging phase. The swinging foot also touches the ground with a flat contact. We consider that the stance leg lifts off the ground after the contact of the swing foot with the ground [6]. 


\subsection{Modeling of the robot biped}

The dynamic modeling is based on the assumption that the stance foot remains fix on the ground, i.e. there is no take off, no sliding and no rotation during the single support phase. As a result, stance foot is considered as base link of the robot biped.

Two models of our biped are used: the first takes into account that the arms are stuck to the trunk such that we consider a seven-link biped; the second considers revolute joints in the shoulders. The generalized coordinates of the biped are described by the vector of absolute angles $\mathbf{q}=\left[\begin{array}{llll}\mathbf{q}_{1} & \mathbf{q}_{2} & \ldots & \mathbf{q}_{\mathrm{n}}\end{array}\right]^{\mathrm{t}} . \boldsymbol{\Gamma}=\left[\begin{array}{ll}\boldsymbol{\Gamma}_{1} & \boldsymbol{\Gamma}_{2}\end{array}\right.$ $\left.\ldots \Gamma_{6}\right]^{\mathrm{t}}$ is the joint torque vector of the locomotor system of the biped. Where $n=6$ or 8 , depending on the biped model.

\subsection{Methodology}

The study is done by the following steps:

\subsubsection{Finding the natural period of arms oscillation:}

We consider the biped arm as a simple pendulum suspended from a frictionless pivot. For small oscillations, the pendulum swings with a natural period about $T_{o} \approx 1.19 \mathrm{sec}$. The natural period depends on the mass, length, and inertia and slightly on initial values of angular variable and angular velocity. The natural period of upper parts oscillation of the biped is chosen in order to coordinate arms motion with the motion of the locomotor system.

\subsubsection{Generation of optimal trajectories for the biped with the arms stuck to the trunk:}

The desired gait is cyclic and consists of a single support phase and an instantaneous impactless flat foot contact with the ground. We use a polynomial of fourth order for the evolution of the actuated joints' motions as a function of time to generate a reference trajectory which minimizes the following criterion:

$$
C_{\Gamma}=\frac{1}{d} \int_{0}^{T} \Gamma^{\mathrm{t}} \boldsymbol{\Gamma} \mathrm{d} t
$$

where $d$ is the step length and $T$ is the time period of one step.

We used the $S Q P$ method (Sequential Quadratic Programming) [8] with the fmincon function of Matlab to solve the parametric optimization problem. Inequality constraints on the ZMP position, the torques and the contact between 
the stance foot and the ground are considered to find the optimal reference trajectory.

\subsubsection{Generation of optimal trajectories with passive movement of the arms:}

Optimal reference trajectories, for the biped with the arms stuck to the trunk, are used for the biped with passive motions of the arms. The upper parts trajectories $\mathbf{q}_{u}(t)$ are due to the dynamic of the locomotor system and are calculated as following:

A system of equations (2) is obtained from the partitioning the matrices of the dynamic model:

$$
\left[\begin{array}{cc}
\mathbf{A}_{l l} & \mathbf{A}_{l u} \\
\mathbf{A}_{u l} & \mathbf{A}_{u u}
\end{array}\right]\left[\begin{array}{c}
\ddot{\mathbf{q}}_{l} \\
\ddot{\mathbf{q}}_{u}
\end{array}\right]+\left[\begin{array}{c}
\mathbf{H}_{l} \\
\mathbf{H}_{u}
\end{array}\right]=\left[\begin{array}{c}
\mathbf{B}_{l} \boldsymbol{\Gamma} \\
0_{2 \times 1}
\end{array}\right]
$$

where the index $u$ refers to the upper parts and the index $l$ refers to the lower parts. $\ddot{\mathbf{q}}_{u} \in R^{2 \times 1}$ is the accelerations vector of the upper parts of the biped, $\ddot{\mathbf{q}}_{l} \in R^{6 \times 1}$ is the accelerations vector of the locomotor system of the biped, $\mathbf{A}_{l l} \in R^{6 \times 6}, \mathbf{A}_{l u} \in R^{6 \times 2}, \mathbf{A}_{u l} \in R^{2 \times 6}$ and $\mathbf{A}_{u u} \in R^{2 \times 2}$ are submatrices of inertia matrix $\mathbf{A} \in R^{8 \times 8}, \mathbf{H}_{u} \in R^{2 \times 1}$ and $\mathbf{H}_{l} \in R^{6 \times 1}$ are submatrices of $\mathbf{H}$ which is a vector of Coriolis, centrifugal and gravity forces. $\mathbf{B}_{l} \in R^{6 \times 6}$ is the actuation matrix.

Then, the upper parts trajectories can be deduced through the numerical integration of the equation (3) with the initial conditions of the angular configurations and velocities of the $\operatorname{arms} \mathbf{q}_{u}(0), \dot{\mathbf{q}}_{u}(0)$ :

$$
\ddot{\mathbf{q}}_{u}=\mathbf{A}_{u u}{ }^{-1}\left(-\mathbf{A}_{u l} \ddot{\mathbf{q}}_{l} \quad-\mathbf{H}_{u}\right)
$$

where $\mathbf{q}_{u}=\left[\mathbf{q}_{7} \mathbf{q}_{8}\right]^{\mathrm{t}}$ (See figure 1$)$.

By taking into account the exchange of the role of the arms between the final time $(t=T)$ of a step and the initial time $(t=0)$ of the next step, the following equalities must be respected to satisfy the cyclicity conditions:

$$
\mathbf{q}_{u}(0)=T_{E} \mathbf{q}_{u}(T), \dot{\mathbf{q}}_{u}(0)=T_{E} \dot{\mathbf{q}}_{u}(T)
$$

where $\mathbf{q}_{u}(0), \dot{\mathbf{q}}_{u}(0)$ vectors of angular and velocities variables of the arms at the initial time $\mathrm{t}=0$ respectively. $\mathbf{q}_{u}(T), \dot{\mathbf{q}}_{u}(T)$ vectors of angular and velocities 
variables of the arms at the final time $\mathrm{t}=T$ respectively. $T_{E}$ is the permutation matrix which does the exchange of the role of arms.

To design the passive motion of the arms, it is necessary to find the solution $\mathbf{q}_{u}(t)$ of system of equation (3) with the boundary values satisfying the equalities (4). A Newton-Raphson method is used to solve the boundary problem. Then, trajectories with the passive motion of the arms can be optimized.

\subsection{Results}

\subsubsection{Time period is constant:}

The time period required such that the arms swing and then return to their initial positions is two times that of the period $T$ of walking step. Therefore, optimal reference trajectories are obtained with a walking period $T$ which is equal to half of the natural period $T_{o}$ of arms oscillation which means $T=T_{o} / 2=0.595 \mathrm{~s}$. By using these reference trajectories for the biped with passive motion of the arms, two possibilities appear depending on initial conditions $\dot{q}_{u}(0), q_{u}(0)$ : a passive motion of the arms with large amplitude and a passive one with very small amplitude which is almost null. A passive motion with large amplitude is possible if and only if the period of walking stride (two steps) is close enough to the natural period of the arms oscillation.

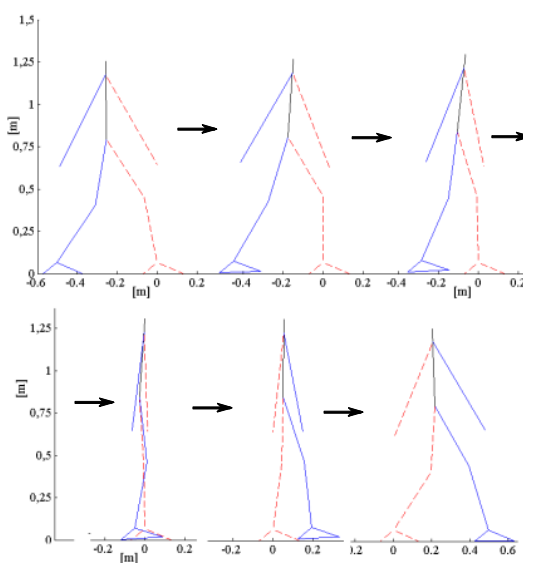

Large amplitude motion

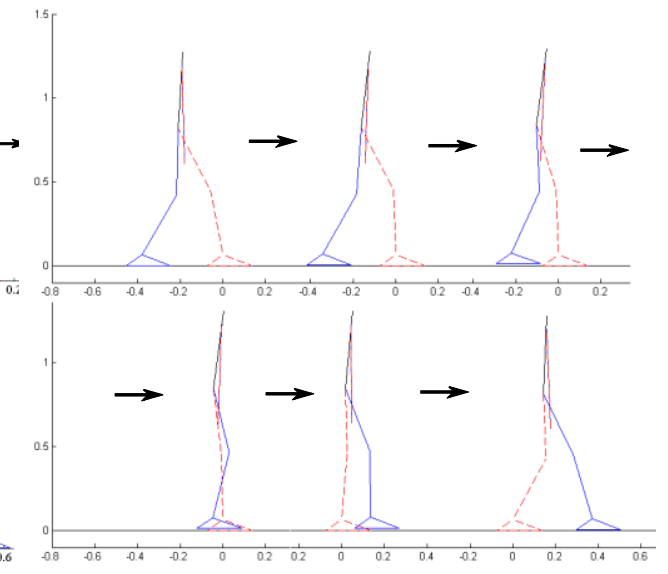

Small amplitude motion

Figure 2. Walking gait as a sequence of snapshot figures is shown at walking speed $\mathrm{V}=0.8 \mathrm{~m} / \mathrm{s}$, 
For walking speed $V=0.8 \mathrm{~m} / \mathrm{s}$, the two types of passive motions are obtained only if the period $T$ is between $0.56 \mathrm{~s}$ and $0.61 \mathrm{~s}$ which is close to $T_{o} / 2$, this interval of $T$ is related to walking speed. If the period $T$ does not belong to the previous interval, only arms motion with small amplitude can be found. Figure 2 shows the two types of passive motion at walking speed of $V=0.8 \mathrm{~m} / \mathrm{s}$ and period $T=0.57 \mathrm{sec}$. In the following, time period is considered as a variable of optimization.

\subsubsection{Time period is a parameter of optimization:}

When reference trajectories are optimized without fixing the time period, optimal time period will be significantly shorter, between $0.35 \mathrm{~s}$ and $0.4 \mathrm{~s}$. Then, passive motions with large amplitude of the arms are not possible. The use of torsion spring in arms joints decreases the natural period and enables to obtain large amplitude of the arms. The time period is used as a parameter of optimization. Then, we found optimum reference trajectories for several walking speeds. Springs' coefficient $k$ is used as a variable of optimization while finding optimal trajectories of the biped with passive motions of the arms. Criterion is calculated for optimal trajectories. Arms passive trajectories with large amplitudes have costs functional lower than these of reference trajectories for all walking speeds, figure 3 .

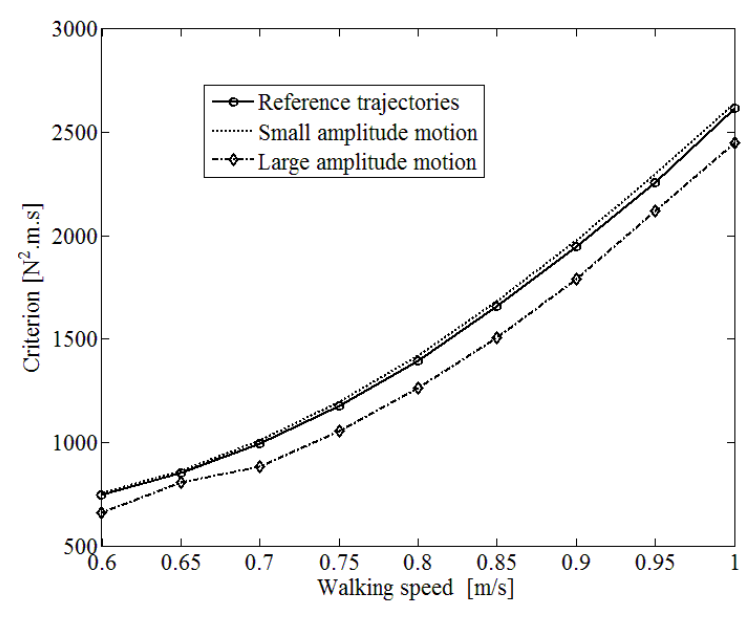

Figure 3. Evolution of energy criterion versus walking speed.

The optimal $k$ is about [8-12] $\mathrm{N} / \mathrm{rad}$ and differs slightly from one walking speed to another. To understand the effect of the springs, we take an optimal trajectory 
obtained at a walking speed $0.75 \mathrm{~m} / \mathrm{s}$ and we changed only the value of the springs' coefficient. The maximum amplitude of arms motion and the cost functional are obtained as functions of $k$. Figure 4, shows that large amplitude of the arms can be obtained when $k=[9.6-13.5] \mathrm{N} / \mathrm{rad}$. Cost functional is minimal when $k$ is equal to $11.3 \mathrm{~N} / \mathrm{rad}$ for a walking speed of $\mathrm{V}=0.75 \mathrm{~m} / \mathrm{s}$. The desired amplitude of arms passive motion can be obtained by changing the springs' coefficient.
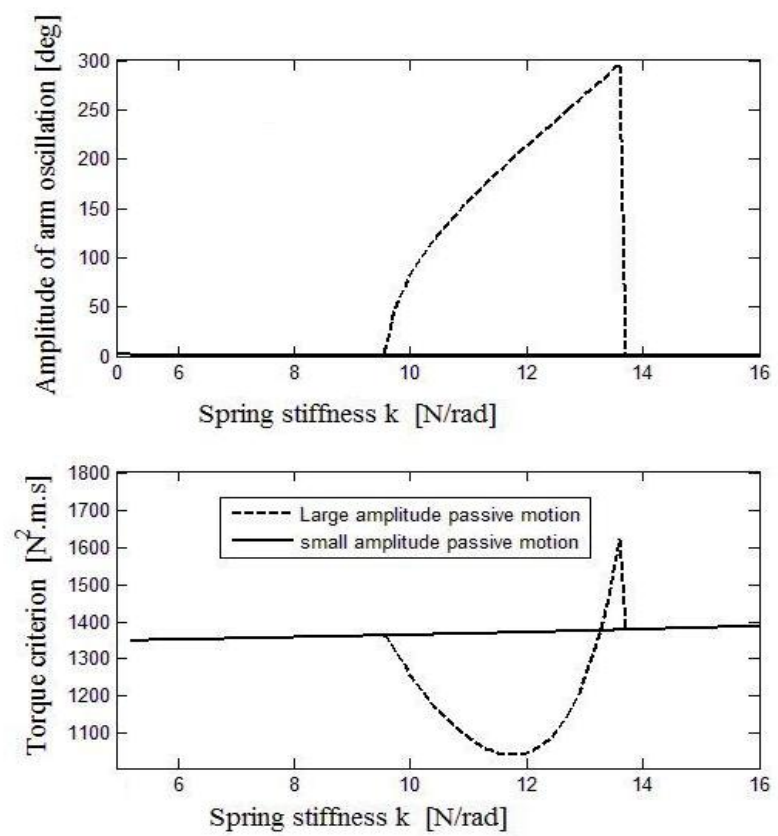

Figure 4. Evolution of energy criterion and maximum amplitude of arms motion versus $k$ at $\mathrm{V}=0.75$ $\mathrm{m} / \mathrm{s}$.

\subsection{Conclusion and Perspectives}

Two types of optimum passive motions of the arms were found during walking of the biped: one with very small amplitude (about one or two degrees) and one with large amplitude. Large amplitude motion can only be obtained if the time period of a step is close enough to half of the natural period of arms oscillation which can be changed by means a torsion spring applied in the arms joints. Cost functional is lower when arms swing with large amplitude passive motion in comparison with both optimal reference trajectories and small amplitude arms motion. It is worth to compare this passive motion with a case where the arms 
are actuated. In this work, we assumed that the swinging leg hits the ground with a flat foot and the support leg immediately leaves the ground. In future works, we study other types of walking gaits having double support phases and impacts besides single support phases such that the swing foot arrives the ground with non flat foot and do a rotation motion until the complete contact with the ground.

\section{References}

1. X. $\mathrm{Mu}$, and $\mathrm{Q}$. Wu, "A complete dynamic model of five-link bipedal walking”. Proceedings of the American Control Conference, 2003, 6, pp. 4926-4931, 2006.

2. D. Tlalolini, Y. Aoustin, and C. Chevallereau, "Design of a walking cyclic gait with single support phases and impacts for the locomotor system of a thirteen-link 3d biped using the parametric optimization". Multibody System Dynamics, 23, pp. 33-56, 2010.

3. C. Chevallereau, and Y. Aoustin, "Optimal reference trajectories for walking and running of a biped robot”. Robotica, 19, pp. 557-569, 2001.

4. S. Kajita, F. Kanehiro, K. Kaneko, K. Fujiwara, K. Harada, K. Yokoi, and H. Hirukawa, "Resolved momentum control: humanoid motion planning based on the linear and angular momentum," International Conference on Intelligent Robots and Systems, vol. 2, pp. 1644 - 1650, Oct. 2003.

5. D. Xing and J. Su, "Arm/trunk motion generation for humanoid robot", SCIENCE CHINA Information Sciences, vol. 53, pp. 1603 - 1612, 2010.

6. Y. Aoustin and A. Formal'skii, "On optimal swinging of the biped arms", International Conference on Intelligent Robots, IROS 2008. pp. 29222927, 2008.

7. S. Alfayad, "Robot humanoïde HYDROïD : Actionnement, Structure Cinématique et Stratégie de contrôle," Thèse de Doctorat de l'Université Versailles Saint Quentin, Paris, Novembre 2009.

8. M. Powell, "Variable metric methods for constrained optimization," Lecture Notes in Mathematics. Springer Berlin / Heidelberg, pp.62-72, 1977. 